\title{
Phonon Transport and Thermoelectricity in Defect-Engineered InAs Nanowires
}

\author{
Annie Weathers ${ }^{1,3}$, Arden L. Moore ${ }^{1,3}$, Michael T. Pettes ${ }^{1,3}$, Daniel Salta ${ }^{2,3}$, Jaehyun Kim ${ }^{1,3}$, \\ Kimberly Dick ${ }^{4,5}$, Lars Samuelson ${ }^{4}$, Heiner Linke ${ }^{4}$, Philippe Caroff ${ }^{6}$, and Li Shi ${ }^{1,2,3}$. \\ ${ }^{1}$ Department of Mechanical Engineering, The University of Texas at Austin, Austin, Texas. \\ ${ }^{2}$ Material Science and Engineering Graduate Program, The University of Texas at Austin, \\ Austin, Texas. \\ ${ }^{3}$ Center for Nano and Molecular Science, The University of Texas at Austin, Austin, Texas. \\ ${ }^{4}$ The Nanometer Structure Consortium (nmC@LU) and Division of Solid State Physics, Lund \\ University, S-22100 Lund, Sweden. \\ ${ }^{5}$ Polymer \& Materials Chemistry, Lund University, S-22100 Lund, Sweden. \\ ${ }^{6}$ Institut d'Electronique, de Microelectronique et de Nanotechnologie, UMR CNRS 8520, \\ F-59652 Villeneuve d'Ascq, France.
}

\begin{abstract}
There have been reports of improvements in the thermoelectric figure of merit through the use of nanostructured materials to suppress the lattice thermal conductivity. Here, we report on a fundamental study of the combined effects of defect planes and surface scattering on phonon transport and thermoelectric properties of defect-engineered InAs nanowires. A microfabricated device is employed to measure the thermal conductivity and thermopower of individual suspended indium arsenide nanowires grown by metal organic vapor phase epitaxy. The four-probe measurement device consists of platinum resistance thermometers and electrodes patterned on two adjacent $\mathrm{SiN}_{\mathrm{x}}$ membranes. A nanowire was suspended between the two membranes, and electrical contact between the nanowire and the platinum electrodes was made with the evaporation of a Ni/Pd film through a shadow mask. The exposed back side of the device substrate allows for characterization of the crystal structure of the suspended nanowire with transmission electron microscopy (TEM) following measurement. The 100-200 nm diameter zincblende (ZB) InAs nanowire samples were grown with randomly spaced twin defects, stacking faults, or phases boundaries perpendicular to the nanowire growth direction, as revealed by transmission electron microscopy (TEM) analysis. Compared to single-crystal ZB InAs nanowires with a similar lateral dimension, the thermal conductivity of the defect-engineered nanowires is reduced by fifty percent at room temperature.
\end{abstract}

\section{INTRODUCTION}

III-V nanowires (NWs) have been investigated as building blocks for electronic and optoelectronic devices, and recently for thermoelectric applications [1-4]. Thermoelectric materials are characterized by their dimensionless figure of merit, $Z T \equiv S^{2} \sigma T / \kappa$, where $S$ is the Seebeck coefficient, $\sigma$ is the electrical conductivity, $\kappa$ is the thermal conductivity, and $T$ is the absolute temperature. There have been efforts to suppress the thermal conductivity by introducing interfaces capable of scattering phonons more efficiently than electrons [5-8]. Thus, the defect planes present in recently reported twinned III-V NWs provide means to control phonon transport. The understanding of phonon transport in III-V NWs with defect planes is important not only for the engineering of thermoelectric materials, but also for evaluating the effects of interfaces and defect structures in heat dissipation in electronic and optoelectronic devices made of NW building blocks. Dhara et al. have shown that the thermal conductivity of InAs NWs with randomly spaced twin planes in the temperature range of $10-50 \mathrm{~K}$ is three orders of magnitude smaller than bulk [9]. However, the self electrical heating method used in 
that work was not able to obtain thermal conductivity at temperatures higher than $50 \mathrm{~K}$ because of a varying temperature coefficient of resistance.

Here we report on the measurement of thermal conductivity and Seebeck coefficient of n-type zincblende (ZB) InAs NWs with randomly spaced twinned planes, stacking faults, or phase boundaries in the temperature range between 80 and $475 \mathrm{~K}$. Compared to single-crystal ZB NWs of a similar lateral dimension in the range of 100-200 nm, the thermal conductivity of the defect-engineered NWs was reduced by about fifty percent, suggesting an apparent effect of the defect planes on phonon transport and thermal conductivity in these NWs.

\section{EXPERIMENT}

InAs NWs were grown by metal-organic vapor phase epitaxy (MOVPE) with trimethylindium (TMIn) and arsine $\left(\mathrm{AsH}_{3}\right)$ precursors, with the NW diameter determined by the size of the gold aerosol seed particles dispersed on the substrate. The NW surface is coated with 5-10 nm of native oxide, which is believed to consist of polycrystalline $\operatorname{In}_{2} \mathrm{O}_{3}$ and $\mathrm{As}_{2} \mathrm{O}_{3}$ [10]. Figure 1(a) shows a scanning electron microscopy (SEM) image of the as-grown vertically orientated NWs on the growth substrate. Figure 1 (b-d) shows high resolution transmission electron microscopy (TEM) images of characteristic features of these NWs. The NWs show two distinct structures along the length of the NWs, both with hexagonal cross sections. Towards the tip of the NW, far from the growth substrate, saw-toothed facets are separated by regularly spaced twin planes [11, 12], shown as the light and dark segments in Fig. 1b. Close to the growth substrate however, the NWs consists of randomly spaced segments also perpendicular to the (111) growth direction but with nearly lateral side walls (Figure 2c-d). The spacing between these segments varies from 2 to $20 \mathrm{~nm}$, which are distinguishable by the alternating dark and light segments. Preliminary diffraction analysis has shown that these randomly spaced segments are zincblende (ZB) InAs. However, further analysis is needed to confirm there are no wurtzite (WZ) phase inclusions in these segments. The diameter varies from approximately $200 \mathrm{~nm}$ close to the growth substrate to $100 \mathrm{~nm}$ at the NW tip.

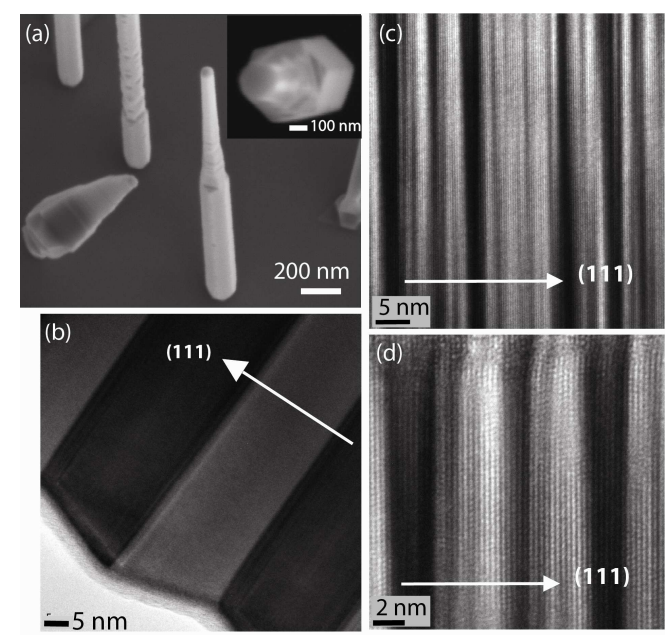

Figure 1. (a) SEM image of vertically aligned NWs with hexagonal cross section (b) TEM image of periodically spaced twin planes found near the NW tip. The 5-10 nm of native oxide is clearly seen. (c-d) TEM image of randomly spaced defect planes found close to the growth substrate. Alternating light and dark segments correspond to alternating twin planes, although further TEM analysis is needed to investigate whether stacking faults or phase boundaries are also present. The defect plane separation ranges between approximately $2-20 \mathrm{~nm}$. 
After growth, the NWs were removed from the substrate by sonicating in methanol, and assembled on a suspended device for measurement, as shown in Figure 2(a-c). The device consists of two adjacent $\operatorname{SiN}_{\mathrm{x}}$ membranes, each supported by six $\operatorname{SiN}_{\mathrm{x}}$ beams. A platinum resistance thermometer (PRT) and two electrodes were patterned on each membrane. The NW was placed across the membrane gap with the help of a tungsten tip attached to a micromanipulator. A separate shadow mask made of a $\operatorname{SiN}_{\mathrm{x}}$ membrane was aligned and attached to the measurement device with the use of a mask aligner and a thin photoresist layer. The $1 \mu \mathrm{m}$ wide windows patterned on the $\mathrm{SiN}_{\mathrm{x}}$ membrane of the shadow mask were aligned to the Pt electrodes. Following the shadow mask assembly, the $\mathrm{NW}$ was cleaned in $\mathrm{O}_{2}$ plasma for 10 seconds to remove any organic residue, and subsequently etched with $\mathrm{BCl}_{3}$ plasma for 20 seconds to remove the native oxide $[13,14]$. The device was then immediately mounted in the vacuum chamber of an electron beam evaporator for depositing $50 \mathrm{~nm} \mathrm{Ni}$ and up to $150 \mathrm{~nm}$ Pd through the windows of the shadow mask onto the NW. The shadow mask was then removed by soaking the assembly in acetone. The NW device was wire bonded to a ceramic chip carrier, and placed in an evacuated continuous flow cryostat for thermal measurement. Figure 2(f) shows the surface of the NW up to $700 \mathrm{~nm}$ from the edge of the deposited metal, which appears to be clean and uncontaminated of evaporated metal, suggesting that metal evaporation through a shadow mask with the sample placed $40 \mathrm{~cm}$ directly above the metal crucible shows minimal spreading of metal outside the shadow mask windows.

After thermal measurements were completed, the crystal structure of the suspended NW over the through-substrate hole in the measurement device was characterized using TEM. Figure 2(d and e) shows the defect planes perpendicular to the (111) direction for NW3 (d) and NW1 (e). However, further work is needed to verify if there are stacking faults or mixed phase $\mathrm{WZ}$ segments in these measured regions in addition to twin planes.
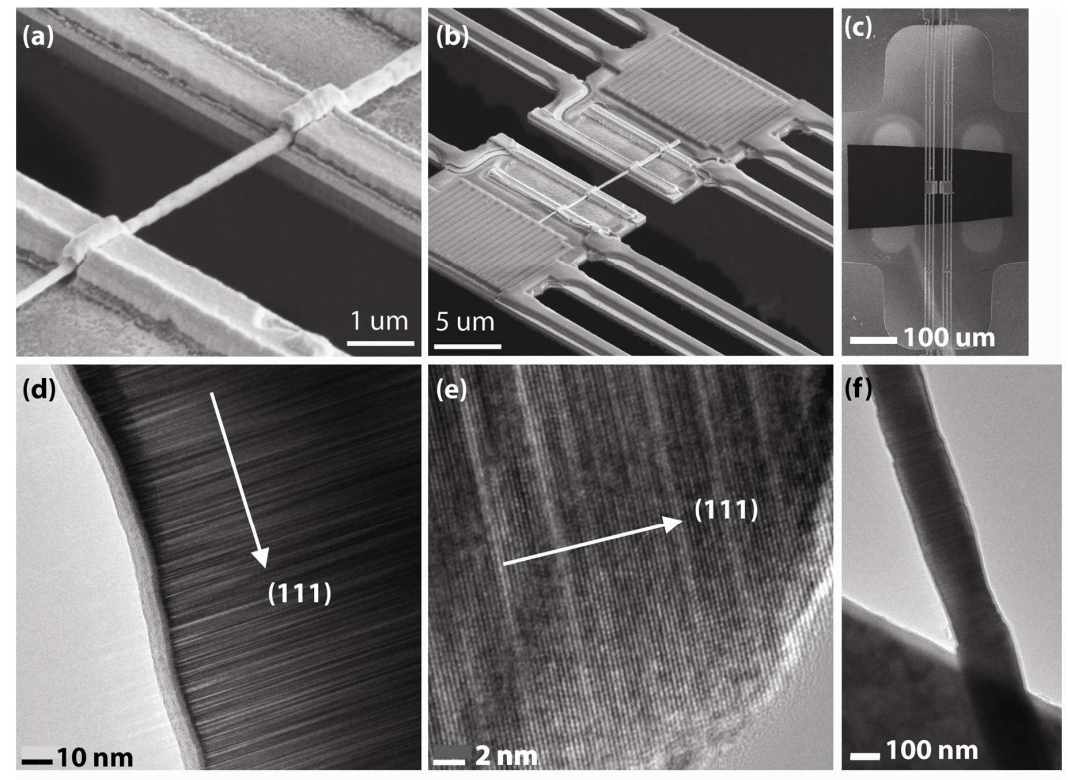

Figure 2. (a-c) SEM image of NW4 on the measurement device after shadow-mask evaporation of Ni/Pd. (d) TEM image of NW3 showing the defect segments perpendicular to the (111) growth direction. (e) TEM image of NW1 and (f) TEM image of NW2 in the vicinity of the metal deposition area. The NW surface appears clean of metal contamination.

During the thermal measurement, a DC voltage provides Joule heating to one PRT membrane. The heat conducted through the sample to the adjacent membrane and the 
temperature rise on the two membranes are measured with the use of the two PRTs, as described in previous publications [15-17]. The thermovoltage was measured across the two inner electrodes (electrodes 2-3 in Figure 3) for NW3, and between electrodes 2-3 and 1-3 for NW4. From the measured conductance of the sample, $G_{s}$, the thermal conductivity $\kappa=$ $L G_{S} / A_{c}$ can be found, where $L$ is the suspended length of the NW and $A$ is the average cross sectional area of the NW, measured with SEM and TEM respectively.

However, due to a finite contact resistance at the NW-membrane interface, the calculated thermal conductance is expected to underestimate the conductivity. It is, however, possible to estimate the thermal contact resistance using a four point Seebeck measurement and a model of the fin temperature profile for the NW. Figure 3 shows the assumed temperature profile along the NW and the definitions of the four temperature points at the heating membrane, sensing membranes and at the edges of the suspended segment. The fin resistance formula gives a contact resistance of $R_{c, i}=\left[\kappa A m \tanh m L_{c, i}\right]^{-1}$, where $\kappa, A, L_{c, i}$, and $m$ are the thermal conductivity, the cross sectional area, and the length of the contact area between the NW and the heating $(i=1)$ and the sensing $(i=2)$ membranes, and $\sqrt{h d / \kappa A}$ respectively, where $h$ is the thermal contact conductance per unit area, and $d$ is the diameter is the NWs. We can derive an expression for the ratio between the inner and outer temperature points [17]

$$
\alpha \equiv \frac{T_{h}-T_{s}}{T_{h}^{\prime}-T_{s}^{\prime}}=1+\frac{1}{L_{s} m}\left[\frac{1}{\tanh m L_{c, 1}}+\frac{1}{\tanh m L_{c, 2}}\right]
$$

where $L_{s}$ is the suspended length, $L_{\mathrm{c}, 1}$ and $L_{\mathrm{c}, 2}$ are the contact lengths on the two membranes. It follows that the thermal resistance of the suspended NW segment is found from

$$
R_{N W}=R_{\text {total }} \alpha
$$

where $R_{\text {total }}$ is the total thermal resistance measured using a two-probe procedure. Hence, we obtain the contact resistance $R_{C}=R_{\text {total }}-R_{N W}$.

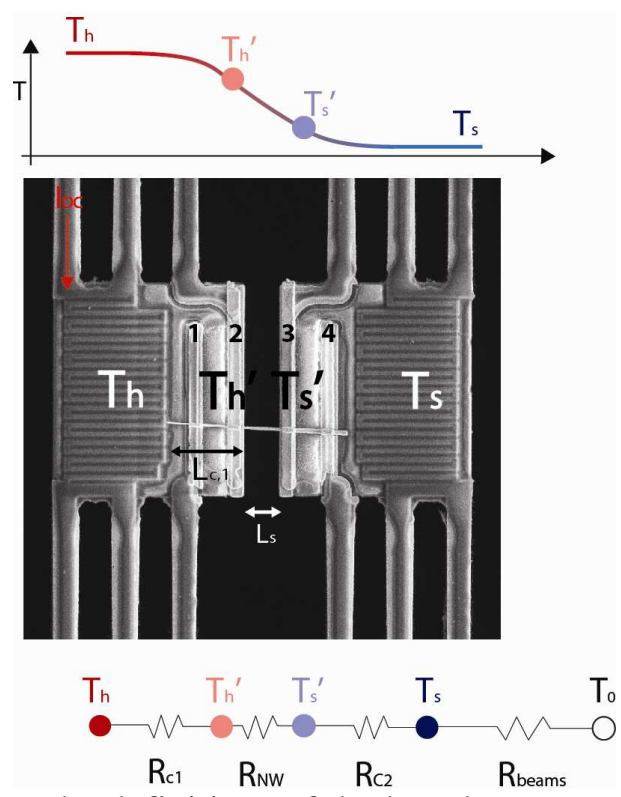

Figure 3. Schematic showing the definitions of the length segments and temperatures used in the four-probe thermal conductivity calculation. 
Complete details of the derivation of the correction factor from the fin resistance is provided in reference [17]. For the three-probe NW4 sample, the contact resistance on each membrane was assumed to be equal, from which a similar analysis can be done. The corrected Seebeck coefficient for NW4 is found from

$$
S=\alpha \frac{V_{23}}{T_{2}-T_{3}}
$$

and is plotted in Figure 4(a) together with the uncorrected, two-probe Seebeck coefficient for NW3. The total measured resistance, the contact resistance, and the corrected sample resistance is plotted in figure 4(b) for NW4. At temperatures below 200K, the contact resistance becomes significant and is found to account for fifty percent of the total measured resistance. The larger uncertainty in NW4 is attributable to the added uncertainty in the calculated contact resistance.
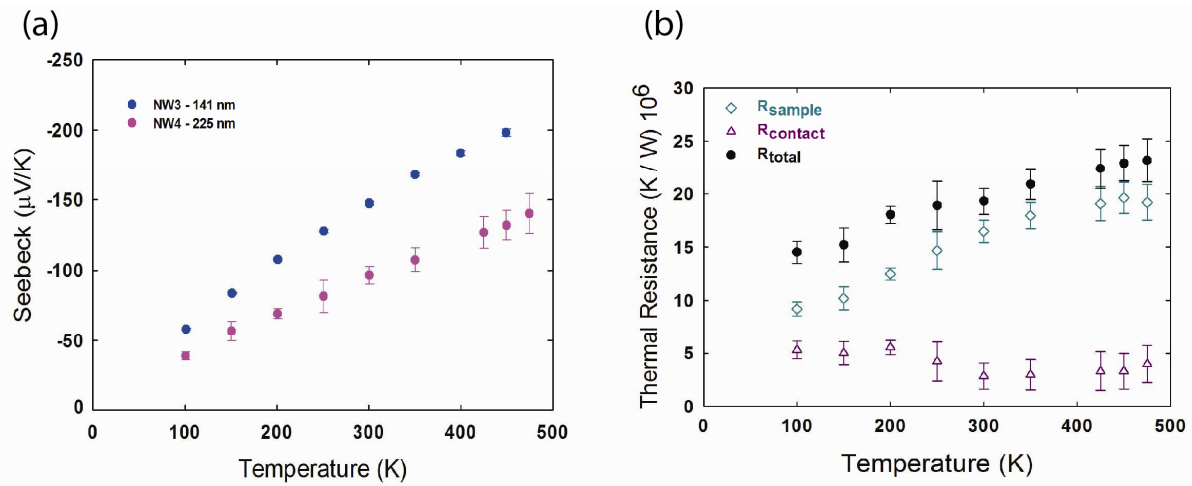

Figure 4. (a) The Seebeck coefficient of NW4 for which the Seebeck coefficient is corrected for the contact resistance (pink), and the two-probe Seebeck coefficient of NW3 measured with the use of the two inner Pt electrodes (blue). (b) The extracted contact thermal resistance, $\mathrm{R}_{\text {contact }}$, and thermal resistance of the sample, $\mathrm{R}_{\text {sample }}$ for NW4. The total measured resistance, $R_{\text {total }}$ is the sum of these two contributions

The measured thermal conductivity of the twinned InAs NWs is shown in Figure 5 together with bulk thermal conductivity values from the literature $[18,19]$ and the single crystal ZB NW data obtained by Zhou et al. [20] using a similar experimental setup. The NWs are assumed to have a hexagonal cross section with the measured diameter assumed to be the average between the edge-to-edge and peak-to-peak lengths of the hexagon. Compared to the single crystal NWs without defect planes, there is a clear reduction in the thermal conductivity by about fifty percent at room temperature. 


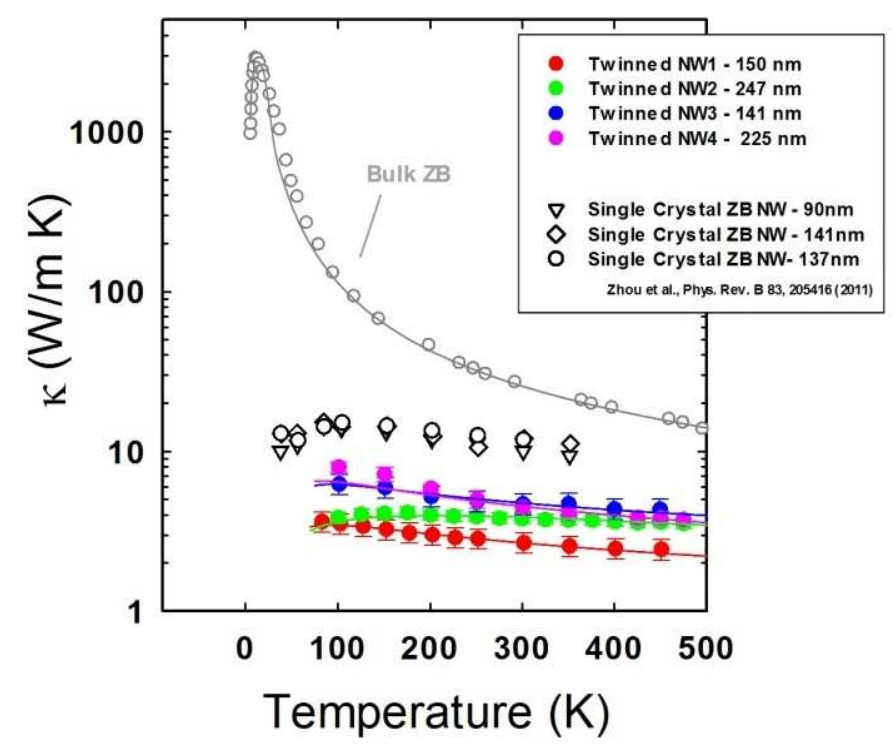

Figure 5. The measured thermal conductivity of twinned, ZB InAs NWs of average diameter indicated in the legend. Single crystal ZB NWs, measured using a similar experimental setup, are shown in black. The dimension values shown in the legend for the single-crystal NWs are the width of one edge of the equilateral triangular cross section of the NW. Bulk ZB InAs is in gray.

The reduction in the thermal conductivity compared to bulk InAs can be explained by enhanced phonon scattering at the twin planes. The thermal conductivity for the twinned NWs can be solved from the discrete phonon dispersion reported by Zhou et al. [20] according to

$$
\kappa=\sum_{s, q} \frac{\Delta q^{3} k_{b}}{8 \pi^{3}} v_{z}^{2} \tau \frac{x^{2} e^{x}}{\left(e^{x}-1\right)^{2}}
$$

where $k_{b}, v_{z}, q, \Delta q^{3}$ are the Boltzmann constant, $z$ component group velocity, phonon wave vector, and discrete volume in the reciprocal space respectively. The sum over $s$ corresponds to a sum over the three acoustic phonon polarizations, $x \equiv \hbar \omega / k_{b} T$, and $\tau$ is the total phonon relaxation time which is assumed to obey Matthiessen's Rule as the sum of the reciprocal relaxation times for all independent scattering processes

$$
\tau^{-1}=\tau_{i m p}^{-1}+\tau_{p h-p h}^{-1}+\tau_{b}^{-1}
$$

The relaxation times are given by $\tau_{i m p}^{-1}=A \omega^{4}$ for impurity scattering, $\tau_{p h-p h}^{-1}=$ $B \omega^{2} T \exp (-C / 3 T)$ for phonon-phonon scattering, and $\tau_{b}^{-1}=v / \ell$ for boundary scattering, with $A, B$, and $C$ as fitting parameters, and $\ell$ as the boundary scattering mean free path (MFP). The contribution to the lattice thermal conductivity from optical modes was assumed to be negligible due to their low group velocity. The bulk InAs thermal conductivity was used to fit the impurity and phonon-phonon scattering terms, and $l$ was adjusted to fit the measured thermal conductivity for the twinned NWs. The results of the model are plotted in figure 5 together with the experimental results. In addition, the extracted boundary scattering mean free path for the four NW samples is plotted in Figure 6 as a function of NW diameter. The extracted MFP is on the order of the twin plane spacing along the length of the NWs, and is an 
order of magnitude lower than the NW diameter. Hence, for these twinned NWs, the boundary scattering is limited not by the NW side walls but by the defect planes.

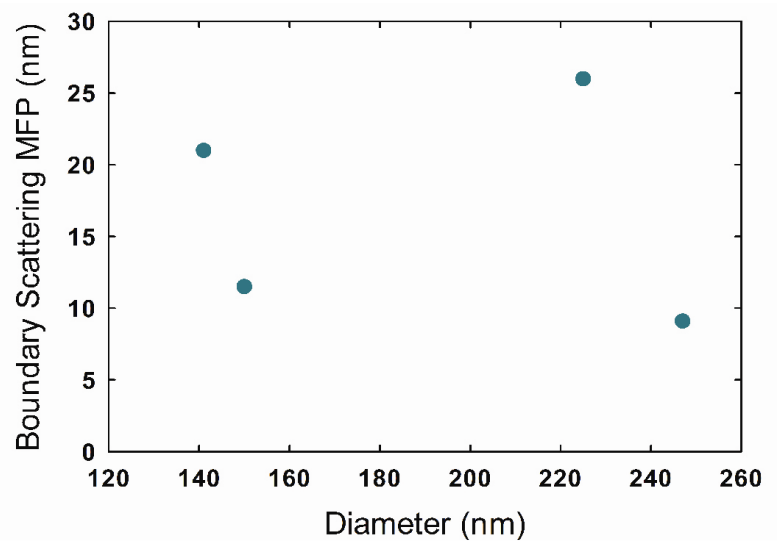

Figure 6. The mean free path of phonons calculated from equation (4) for the four NW samples.

\section{CONCLUSION}

The thermal conductivity and Seebeck coefficient of suspended InAs NWs with randomly spaced defect planes were measured in the temperature range of $100-475 \mathrm{~K}$. The thermal conductivity shows a fifty percent reduction compared to single crystal ZB NWs with a similar lateral dimension. The phonon-boundary scattering mean free path is determined from the measured thermal conductivity to be on the order of $10 \mathrm{~nm}$, comparable to the separation between defect planes found from the TEM analysis of the sample, and smaller than the $\sim 150$ $\mathrm{nm}$ diameter of the NW. The results provide insight into the phonon transport in defect-engineered NWs for both thermoelectric and electronic device applications. However, further four-probe electrical conductivity measurements of the NWs are needed to quantify the effects of twin planes on the electrical conductivity of ZB NWs [21, 22].

\section{ACKNOWLEDGEMENTS}

This work was supported by the National Science Foundation (NSF) Thermal Transport Processes program, the Office of Naval Research, the NSF Graduate Research Fellowship Program, the Swedish Energy Agency (Energimyndigheten), nmC@LU, the Swedish Research Council and the Knut and Alice Wallenberg Foundation.

\section{REFERENCES}

1. M. Gudiken, L. Lauhon, J. Wang, D. Smith and C. M. Lieber, Nature 409, 617 (2002).

2. C. Fasth, A. Fuhrer, L. Samuelson, V. N. Golavach and D. Loss, Physical Review Letters 98 , 266801 (2007).

3. N. Mingo, Applied Physics Letters 84 14, 2652 (2004).

4. X. Duan, Y. Huang, Y. Cui, J. Wang and C. M. Lieber, Nature 409 , 66 (2001).

5. Y. Pei, N. A. Heinz, A. LaLonde and G. J. Snyder, Energy and Environmental Science 4, 3640 (2011).

6. A. J. Minnich, M. S. Dresselhaus, Z. F. Ren and G. Chen, Energy and Environmental Science 2, 466 (2009).

7. Y. K. Koh, C. J. Vineis, S. D. Calawa, M. P. Walsh and D. G. Cahill, Applied Physics 
Letters 94,153101 (2009).

8. C. J. Vineis, A. Shakouri, A. Majumdar and M. G. Kanatzidis, Advanced Materials 22, 3970 (2010).

9. S. Dhara, H. S. Solanki, A. P. R., V. Singh, S. Sengupta, B. A. Chalke, A. Dhar, M. Gokhale, A. Bhattacharya and M. M. Deshmukh, Physical Review B 84, 121307 (2011).

10. G. Hollinger, R. Skheyta-Kabbani and M. Gendry, Physical Review B 49 16, 11159 (1994).

11. P. Caroff, K. A. Dick, J. Johansson, M. E. Messing, K. Deppert and L. Samuelson, Nature Nanotech 4, 50 (2009).

12. C. Thelander, K. A. Dick, M. T. Borgstrom, L. E. Froberg, P. Caroff, H. A. Nilsson and L. Samuelson, Nanotechnology 21 14, 205703 (2010).

13. M. N. Kutty, E. Plis, A. Khoshakhlagh, S. Myers, N. Gautam, S. Smolev, Y. D. Sharma, R. Dawson, S. Krishna, S. J. Lee and S. K. Noh, Journal of Electronic Materials 3910 , 2203 (2010).

14. G. Smolinsky, R. P. Chang and T. M. Mayer, Journal of Vacuum Science and Technology 18 1, 12 (1981).

15. C. Yu, S. Saha, J. Zhou, L. Shi, A. Cassell, B. A. Cruden, Q. Ngo and J. Li, Journal of Heat Transfer 128,235 (2006).

16. L. Shi, D. Li, C. Yu, W. Jang, D. Kim, Z. Yao, P. Kim and A. Majumdar, Journal of Heat Transfer 125,881 (2003).

17. A. Mavrokefalos, M. T. Pettes, F. Zhou and L. Shi, Review of Scientific Instruments 78, 034901 (2007).

18. R. Bowers, W. Ure, J. E. Bauerle and A. J. Cornish, Journal of Applied Physics 306 , 930 (1959).

19. G. L. Guillou and H. J. Albany, Physical Review B 5 6, 2301 (1972).

20. F. Zhou, A. L. Moore, J. Bolinsson, L. Froberg, M. T. Pettes, H. Kong, L. Rabenberg, P. Caroff, D. A. Stewart, N. Mingo, K. A. Dick, L. Samuelson, H. Linke and L. Shi, Physical Review B 83, 205416 (2011).

21. C. Thelander, P. Caroff, S. Plissard, A. W. Dey and K. A. Dick, Nano Letters 11, 2424 (2011).

22. M. D. Schroer and J. R. Petta, Nano Letters 10, 1618, (2010). 